\title{
Optimal control for quadrotors during inspection of power utility assets
}

\author{
A. Ailon \\ Department of Electrical and Computer Engineering \\ Ben Gurion University of the Negev, Beer Sheva 8410501 (Israel) \\ and School of Electrical Engineering \\ Afeka Academic College of Engineering, Tel Aviv 6910717 (Israel) \\ Phone number: +972 54 6602828, e-mail: Amit@ee.bgu.ac.il
}

\begin{abstract}
Renewable energy and power utilities inspection by autonomous aircraft enables rapid and effective risk-free assessment of the state of systems, and provides a qualitative and accurate assessment of defects and damages. To realize maximum operational benefits of aerial inspections, effective controls for autonomous aircraft must be ensured and the system should be operated in an optimal policy. Hence, the objective of this paper is to consider optimal control strategies for a quadrotor helicopter type UAV. Based on some structural properties of the considered system, in particular the flatness property, we suggest a control strategy that ensures tracking a time parameterized path that connects two given points in the state space while minimizing the energy consumption. The proposed controller enables longer-endurance missions for the quadrotor and effective supervision and inspection of large energy systems and power plants. In this paper the analysis of the control law takes into consideration the highly nonlinear dynamic model of the quadrotor and the electrical actuator model. The current approach in solving the optimal control problem under consideration allows one to eliminate the differential equation and to reformulate the optimal control problem to a nonlinear dynamic programming problem. The approach can make monitoring operations of renewable energy plants by aerial vehicles, more efficient.
\end{abstract}

\section{Key words}

Aerial inspections for power utilities, quadrotors, nonlinear systems, optimal control, minimum energy.

\section{Introduction}

Use of drones has had a tremendous impact on inspection of civil infrastructures including, buildings, bridges, power lines, solar and wind turbine farms [1], [2], [3]. The use of drones in the field of renewable energy is demonstrated in tasks such as designing power plants, monitoring hardware of power lines and renewable systems. In recent years wind turbines have gotten taller and wind blades and rotors have gotten bigger [4] and the need for efficient and accurate surveillance and inspection by drones has become more significant. Infrared cameras mounted on drones can help detect malfunctions in solar panels and enable faster repairs [5]. In the field of wind turbines, companies can deploy drones to get close-up 3D images of wind blades without stopping the turbine operation. Hence, the need arises to develop effective control laws for UAVs that will ensure optimal performance, maneuverability, and stability of the aircraft. We concentrate on the minimum energy control problem in a quadrotor UAV. In Fig. 1 we present a scheme of the quadrotor with the four electric motors and their functionality as it expressed by their relative direction of rotation.



Figure 1. A camera-equipped quadrotor. The quadrotor is controlled by varying the motor relative speeds $\Omega_{i}$.

Several papers have considered optimal control problems in quadrotors. For example [6] considers $L_{1}$ - optimal robust controller for the quadrotor UAV, and [7] and [8] have studied the optimal control problems when the objective is to minimize the system energy consumption,

In [12], the flatness property is used in solving an optimal control problem. The contribution of this article is in applying the concept of flatness in a model that accounts for the full nonlinear model, including the motor dynamics. We establishe a control law that brings the UAV to a desired final state from a given state within a prescribed time with minimum expenditure of energy.

\section{Modelling}

The quadrotor and the earth frames are given in in Fig. 2, where $\mathrm{C}$ designates the UAV center of mass. Let $\xi=[\phi, \theta, \psi]^{T}$ be the Euler angles vector. Then, the rotation matrix $\mathbb{R}(\xi)$ that translates the quadrotor-fixed frame quantities into the earth-fixed frame is given by (we use the familiar abbreviations $S_{\alpha} \doteq \sin \alpha, C_{\alpha} \doteq \cos \alpha$ ) 


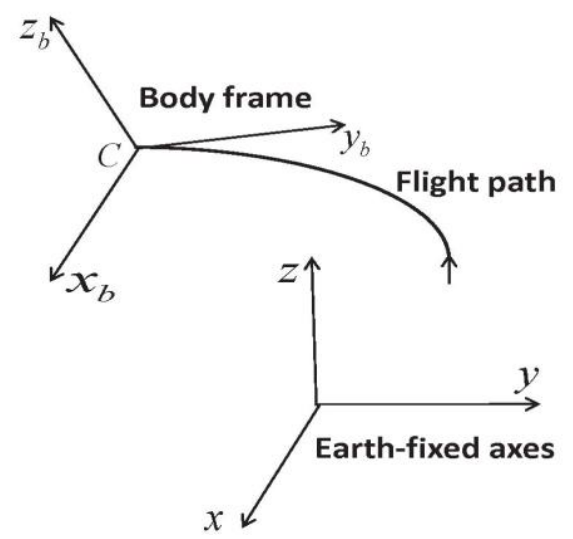

Figure 2. The earth-fixed $\&$ body frames. $C$ is the mass center.

$$
\mathbb{R}(\xi)=\left[\begin{array}{ccc}
C_{\theta} C_{\psi} & C_{\psi} S_{\theta} S_{\phi}-C_{\phi} S_{\psi} & C_{\psi} S_{\theta} C_{\phi}+S_{\phi} S_{\psi} \\
C_{\theta} S_{\psi} & C_{\phi} C_{\psi}+S_{\theta} S_{\phi} S_{\psi} & -S_{\phi} C_{\psi}+S_{\theta} C_{\phi} S_{\psi} \\
-S_{\theta} & C_{\theta} S_{\phi} & C_{\theta} C_{\phi}
\end{array}\right]
$$

Then, provided $\theta \in(-\pi / 2, \pi / 2)$ using the Lagrange formulation the system nonlinear dynamical model is [9]: $\dot{\varphi}_{1}=\varphi_{2}$

$\dot{\varphi}_{2}=\mathbb{R}(\xi) F_{b} / m-e_{3} g$

$\dot{\xi}=L(\xi) \omega$

$\dot{\omega}=J^{-1} S(J \omega) \omega+J^{-1} M_{b}$

where $\varphi_{1}=[x, y, z]^{T}, \omega$ is the quadrotor angular velocity (in the body frame), $m, J$ are respectively the mass and moment of inertia, $F_{b}, M_{b}$ are respectively the thrust and the torque, $S(J \omega) \omega=(J \omega) \times \omega$ (the cross product), $e_{3}=[0,0,1]^{T}$ and $g$ is the gravity acceleration.

For $\theta \in(-\pi / 2, \pi / 2)$ the matrix $L(\xi)$ in (2) is given by $L(\xi)=\left[\begin{array}{ccc}1 & S_{\phi} T_{\theta} & C_{\phi} T_{\theta} \\ 0 & C_{\phi} & -S_{\phi} \\ 0 & S_{\phi} / C_{\theta} & C_{\phi} / C_{\theta}\end{array}\right]$

The thrust force provided by the $i-$ th motor is $f_{i}=b \Omega_{i}{ }^{2}$ where $b>0$ is the thrust constant and $M_{b}=\left[l b\left(\Omega_{1}{ }^{2}-\Omega_{3}{ }^{2}\right), l b\left(\Omega_{4}{ }^{2}-\Omega_{2}{ }^{2}\right), d\left(\Omega_{1}{ }^{2}+\Omega_{3}{ }^{2}-\Omega_{2}{ }^{2}-\Omega_{4}{ }^{2}\right)\right]$

$F_{b}=\left[0,0, U_{b}\right]^{T}, U_{b}=b \sum_{1}^{4} \Omega_{i}^{2}$

where $\Omega_{i}$ is the rotor angular velocity, $l$ is the distance between the center of mass (the point $C$ in Fig.2) and the rotor shaft, $d>0$ is the drag factor.

Next, we consider the actuator dynamics. We assume four identical brushless direct current (BLDC) motors [8]. A simplified model of the system (motor \& load) is given by

$$
\begin{aligned}
& v_{i}(t)=R i(t)+\operatorname{Li}(t)+\Omega_{i}(t) / k_{v} \\
& I \dot{\Omega}_{i}(t)=\tau(t)-d \Omega_{i}{ }^{2}(t)-d_{v} \Omega_{i}(t)
\end{aligned}
$$

where $R, L$ are the motor resistance and inductance, $\tau(t)=k_{t} i(t)$ is the motor torque, $k_{v}, k_{t}$ are constants, $d_{v} \Omega_{i}(t)$ is a torque due to viscous friction, $I<<J$ is the rotor with the propeller inertia moment.

\section{The Flight Control Objectives}

The energy consumed by the quadrotor during a time interval $\left[0, t_{f}\right]$ is given by $[8]$ :

$$
E_{f}=\int_{0}^{t_{f}} \sum_{i=1}^{4} \tau_{i}(t) \Omega_{i}(t) d t
$$

or, equivalently (using (5))

$$
E_{f}=\int_{0}^{t_{f}} \sum_{i=1}^{4}\left(I \dot{\Omega}_{i}(t) \Omega_{i}(t)+d \Omega_{i}^{3}(t)+d_{v} \Omega_{i}{ }^{2}(t)\right) d t
$$

The objective is to establish a control strategy such that the following is satisfied. For a given initial state of the system (2) and for a desired final target at $t=t_{f}>0$ in the state-space the trajectory connecting the starting and ending points is selected such that the energy consumption is minimal, namely, $E_{f} \rightarrow \min$.

Practically, the current approach ensures a suboptimal solution. In this paper we apply the so-called flatness property in the theory of nonlinear systems [10].

\section{Applying the Flatness Property}

To apply the flatness property in the quadrotor nonlinear model [9], [11], [12] we recall that the flat output is given by the following vector-valued function:

$$
y_{f}{ }^{*}(t)=\left[\varphi_{1}^{* \mathrm{~T}}(\mathrm{t}), \psi^{*}(t)\right]^{T}=\left[x^{*}(\mathrm{t}), y^{*}(\mathrm{t}), z^{*}(\mathrm{t}), \psi^{*}(\mathrm{t})\right]^{T}
$$

We will represent $y_{f}{ }^{*}(t)$ by a linear combination of a set of linearly independent smooth functions with undetermined coefficients. We will select the coefficients such that a set of given conditions are met and along a computed trajectory $E\left(t_{f}\right) \rightarrow \min$. To simplify slightly the procedure, we assume that $\psi(0)=\psi\left(t_{f}\right)=0$ and $\psi^{*}(t)=0$ for all $0 \leq t \leq t_{f}$.

Hence, from (8) it remains to $\operatorname{select} \varphi_{1}^{*}(t)$ that determines the trajectory and the input functions. To achieve this goal, we need first to show the mathematical relationships between the flat output and the system variables (state and inputs).

Firstly, we list a set of conditions that must be satisfied by the selected flat output: (i)- $\ddot{z}^{*}(t)+\mathrm{g}>0$ (free fall is not allowed), (ii)- the computed thrust must be strictly positive, that is $U_{b}{ }^{*}(t)>0$, and (iii)- the computed pair $-\pi / 2<\phi^{*}(t), \theta^{*}(t)<\pi / 2$ for all $t \geq 0$. 
To slightly simplify notations, assume without loss of generality that $m=1$. From (1), (2), and (4) we have

$$
\mathbb{R}^{*} F_{b}^{*}=\left[\begin{array}{c}
C_{\psi^{*}} S_{\theta^{*}} C_{\phi^{*}}+S_{\phi^{*}} S_{\psi^{*}} \\
-S_{\phi^{*}} C_{\psi^{*}}+S_{\theta^{*}} C_{\phi^{*}} S_{\psi^{*}} \\
C_{\theta^{*}} C_{\phi^{*}}
\end{array}\right] U_{b}^{*}
$$

Summarizing the squares of the expressions in the matrix:

$\left(C_{\psi^{*}} S_{\theta^{*}} C_{\phi^{*}}+S_{\phi^{*}} S_{\psi^{*}}\right)^{2}+\left(-S_{\phi^{*}} C_{\psi^{*}}+S_{\theta^{*}} C_{\phi^{*}} S_{\psi^{*}}\right)^{2}+\left(C_{\theta^{*}} C_{\phi^{*}}\right)^{2}=$ $C_{\psi^{*}}{ }^{2} S_{\theta^{*}}{ }^{2} C_{\phi^{*}}{ }^{2}+S_{\phi^{*}}{ }^{2} S_{\psi^{*}}{ }^{2}+S_{\phi^{*}}{ }^{2} C_{\psi^{*}}{ }^{2}+S_{\theta^{*}}{ }^{2} C_{\phi^{*}}{ }^{2} S_{\psi^{*}}{ }^{2}+C_{\theta^{*}}{ }^{2} C_{\phi^{*}}{ }^{2}=1$ and recalling (2) $U_{b}^{* 2}=\ddot{x}^{* 2}+\ddot{y}^{* 2}+\left(\ddot{z}^{*}(t)+\mathrm{g}\right)^{2}$ or

$U_{b}^{*}(t)=m \sqrt{\ddot{x}^{*}(t)^{2}+\ddot{y}^{*}(t)^{2}+\left(\ddot{z}^{*}(t)+\mathrm{g}\right)^{2}}>0$

Since $\ddot{z}^{*}(t)+\mathrm{g}>0$ the strict inequality in (9) holds.

Recalling that we select $\psi^{*}(t)=0$, from (2) we have $\ddot{x}^{*} / U_{b}^{*}=S_{\theta^{*}} C_{\phi^{*}}, \ddot{y}^{*} / U_{b}^{*}=-S_{\phi^{*}}$ and thus

$\phi^{*}=-\arcsin \left(\ddot{y}^{*} / \sqrt{\ddot{x}^{* 2}+\ddot{y}^{* 2}+\left(\ddot{z}^{*}(t)+\mathrm{g}\right)^{2}}\right)$

$\theta^{*}=\arcsin \left(\ddot{x}^{*} / \sqrt{\ddot{x}^{* 2}+\left(\ddot{z}^{*}(t)+\mathrm{g}\right)^{2}}\right)$

Remark. By (9) \& $\ddot{z}^{*}(t)+\mathrm{g}>0$ we have $\left|\ddot{y}^{*} / U_{b}^{*}\right|<1$, $\left|\ddot{x}^{*} / U_{b}^{*}\right|<1$ and therefore $\theta \in(-\pi / 2, \pi / 2)$.

Thus far we have determined by the flat output (8) the quadrotor position coordinate $\varphi_{1}^{*}=\left[x^{*}, y^{*}, z^{*}\right]^{T}$, the Euler angles vector $\xi^{*}=\left[\phi^{*}, \theta^{*}, \psi^{*}\right]^{T}$, and the thrust $U_{b}^{*}$. Therefore, we have $\varphi_{2}^{*}=\dot{\varphi}_{1}^{*}=\left[\dot{x}^{*}, \dot{y}^{*}, \dot{z}^{*}\right]^{T}$ and $\dot{\xi}^{*}=\left[\dot{\phi}^{*}, \dot{\theta}^{*}, \dot{\psi}^{*}\right]^{T}$. In $\theta \in(-\pi / 2, \pi / 2)$ the inverse of $L(\xi)$ exists and letting $L^{-1}(\xi) \doteq Q(\xi)$ we have by (2)

$\omega^{*}=Q\left(\xi^{*}\right) \dot{\xi}^{*}=\left[\begin{array}{ccc}1 & 0 & -S_{\theta^{*}} \\ 0 & C_{\phi^{*}} & C_{\theta^{*}} S_{\phi^{*}} \\ 0 & -S_{\phi^{*}} & C_{\theta^{*}} C_{\phi^{*}}\end{array}\right] \dot{\xi}^{*}$

which determines $\omega^{*}(t)$ and hence $\dot{\omega}^{*}(t)$. Applying $\omega^{*}(t)$ and $\dot{\omega}^{*}(t)$ we get from the last equation of (2)

$M_{b}^{*}=J \dot{\omega}^{*}-S\left(J \omega^{*}\right) \omega^{*}$

Hence, the above procedure yields the chain of results: $y_{f}^{*} \rightarrow \varphi_{1}^{*} \rightarrow \varphi_{2}^{*} \rightarrow U_{b}^{*} \rightarrow \xi^{*} \rightarrow \omega^{*} \rightarrow M_{b}^{*}$

\section{Some Computational Considerations}

The initial and final times are $t_{0}=0, t_{f}>0$ respectively. The quadrotor initial and final states are: $\varphi_{1}(0)=\varphi_{10}, \varphi_{2}(0)=\varphi_{20}, \xi(0)=\xi_{0}, \omega(0)=\omega_{0}$ $\varphi_{1}\left(t_{f}\right)=\varphi_{1 f}, \varphi_{2}\left(t_{f}\right)=\varphi_{2 f}, \xi\left(t_{f}\right)=\xi_{f}, \omega\left(t_{f}\right)=\omega_{f}$

To simplify a bit the presentation we assume that $\varphi_{1}(0)=0, \varphi_{2}(0)=0, \xi(0)=0, \omega(0)=0$

$\varphi_{1}\left(t_{f}\right)=\varphi_{1 f}, \varphi_{2}\left(t_{f}\right)=0, \xi\left(t_{f}\right)=0, \omega\left(t_{f}\right)=0$

That is, we search for an optimal control strategy that transfers the UAV from rest to an arbitrary selected point $\left\{x_{f}, y_{f}, z_{f}\right\}$ with zero linear and angular velocities and the initial and final orientation are $\xi(0)=\xi\left(t_{f}\right)=0$.

As indicated the flat output in (8) can be selected as a linear combination of linearly independent functions. For illustration, we take polynomial functions (which, numerically is not the best choice) for the position components of the flat output, namely,

$$
x^{*}(t)=\sum_{i=0}^{n_{x}} a_{i} t^{i}, y^{*}(t)=\sum_{i=0}^{n_{y}} b_{i} t^{i}, z^{*}(t)=\sum_{i=0}^{n_{z}} c_{i} t^{i}
$$

where $n_{x}, n_{y}, n_{z}$ must be chosen large enough such that the listed conditions hold and $E_{f} \rightarrow$ min., namely,

$$
\begin{aligned}
& \varphi_{1}^{*}(0)=0, \varphi_{2}^{*}(0)=0, \xi^{*}(0)=0, \omega^{*}(0)=0 \\
& \varphi_{1}^{*}\left(t_{f}\right)=\varphi_{1 f}, \varphi_{2}^{*}\left(t_{f}\right)=0, \xi^{*}\left(t_{f}\right)=0, \omega^{*}\left(t_{f}\right)=0
\end{aligned}
$$

where $\xi^{*}\left(t_{f}\right)=\left[\phi_{f}^{*}, \theta_{f}^{*}, 0\right]^{T}, \phi_{f}^{*}=\theta_{f}^{*}=0$ are given in (10), and $\omega^{*}\left(t_{f}\right)$ in (11). In addition, we recall that $\ddot{z}^{*}(t)+\mathrm{g}>0$ must hold. Next, $E_{f}$ in (7) will be presented as a function of the flat output. From (4):

$\left[\begin{array}{c}U_{b}^{*} \\ M_{b}^{*}\end{array}\right]=\left[\begin{array}{cccc}b & b & b & b \\ l b & 0 & -l b & 0 \\ 0 & -l b & 0 & l b \\ d & -d & d & -d\end{array}\right]\left[\begin{array}{l}\Omega_{1}^{* 2} \\ \Omega_{2}^{* 2} \\ \Omega_{3}^{* 2} \\ \Omega_{4}^{* 2}\end{array}\right] \doteq K \Gamma^{*}$

where $\Gamma^{*}=\left[\Omega_{1}^{* 2}, \Omega_{2}{ }^{* 2}, \Omega_{3}{ }^{* 2}, \Omega_{4}{ }^{* 2}\right]^{T}$ and the constant matrix $K$ in (19) is invertible $\left(\operatorname{det}(K)=8 b^{3} l^{2} d\right)$. So, $\left[\begin{array}{l}\Omega_{1}{ }^{{ }_{2}} \\ \Omega_{2}{ }^{* 2} \\ \Omega_{3}{ }^{{ }_{2}} \\ \Omega_{4}{ }^{{ }_{2}}\end{array}\right]=K^{-1}\left[\begin{array}{c}U_{b}{ }^{*} \\ M_{b}{ }^{*}\end{array}\right] \rightarrow\left[\begin{array}{l}\Omega_{1}{ }^{*} \dot{\Omega}_{1}{ }^{*} \\ \Omega_{2}{ }^{*} \dot{\Omega}_{2}{ }^{*} \\ \Omega_{3}{ }^{*} \dot{\Omega}_{3}{ }^{*} \\ \Omega_{4}{ }^{*} \dot{\Omega}_{4}{ }^{*}\end{array}\right]=\frac{1}{2} K^{-1}\left[\begin{array}{c}\dot{U}_{b}{ }^{*} \\ \dot{M}_{b}{ }^{*}\end{array}\right]$

where $\dot{U}_{b}{ }^{*}$ is defined by (9) and $\dot{M}_{b}{ }^{*}$ by (12), that is 
$\dot{M}_{b}^{*}=J \ddot{\omega}^{*}-\left(J \dot{\omega}^{*}\right) \times \omega^{*}-\left(J \omega^{*}\right) \times \dot{\omega}^{*}$. Hence, the expressions that appear in the integrant of (7) can be displayed using the starred functions in (16).

Some immediate results are as follows. From (10) and (14)-(15) we obtain the following chain: $\xi(0)=\xi\left(t_{f}\right)=0 \rightarrow \ddot{x}(0)=\ddot{x}\left(t_{f}\right)=\ddot{y}(0)=\ddot{y}\left(t_{f}\right)=0$.

But then we also have (see (10)-(11)) $\dot{\xi}(0)=\dot{\xi}\left(t_{f}\right)=0 \rightarrow x^{(3)}(0)=x^{(3)}\left(t_{f}\right)=y^{(3)}(0)=y^{(3)}\left(t_{f}\right)=0$.

It is also reasonable to assume that the initial and final angular accelerations and the initial and final vertical accelerations are zeros (assuming that in aerial photo missions, when taking a picture, the camera should be held steady), i.e. $\dot{\omega}(0)=\dot{\omega}\left(t_{f}\right)=0$ and $\ddot{z}(0)=\ddot{z}\left(t_{f}\right)=0$.

Then $\quad \dot{\omega}(0)=\dot{\omega}\left(t_{f}\right)=0 \rightarrow \ddot{\xi}(0)=\ddot{\xi}\left(t_{f}\right)=0 \quad$ and $\ddot{\xi}(0)=\ddot{\xi}\left(t_{f}\right)=0 \rightarrow x^{(4)}(0)=x^{(4)}\left(t_{f}\right)=\mathrm{y}^{(4)}(0)=y^{(4)}\left(t_{f}\right)=0$.

Therefore, the following set of equations must hold:

$\sum_{i=5}^{n_{x}} a_{i} t_{f}^{i}=x_{1}\left(t_{f}\right) ; \frac{d^{j}}{d t^{j}} \sum_{i=5}^{n_{x}} a_{i} t_{f}^{i}=0, j=1,2,3,4$

$\sum_{i=5}^{n_{x}} b_{i} t_{f}{ }^{i}=y\left(t_{f}\right) ; \frac{d^{j}}{d t^{j}} \sum_{i=5}^{n_{x}} b_{i} t_{f}{ }^{i}=0, j=1,2,3,4$

$\sum_{i=5}^{n_{x}} c_{i} t_{f}{ }^{i}=z\left(t_{f}\right) ; \frac{d^{j}}{d t^{j}} \sum_{i=5}^{n_{x}} c_{i} t_{f}{ }^{i}=0, j=1,2$

With these results in mind, we can formulate the current optimal control problem as follows. Find a set of coefficients $a_{i}, b_{i}, c_{i}$ of the polynomials

$$
x^{*}(t)=\sum_{i=5}^{n_{x}} a_{i} t^{i}, y^{*}(t)=\sum_{i=5}^{n_{y}} b_{i} t^{i}, z^{*}(t)=\sum_{i=3}^{n_{3}} c_{i} t^{i}
$$

such that (21) holds, $\ddot{z}^{*}(t)+\mathrm{g}>0$, and $E_{f} \rightarrow$ min.

\section{A Sub-Optimal Control Strategy}

In the considered quadrotor each motor rotates in one direction: either clockwise with respect to the positive direction of the $Z_{b}$ axis in Fig. 1 (motors 1 and 3) or counterclockwise (motors 2 and 4). So, when we calculate the energy invested per each motor, we regard $\Omega_{i}{ }^{*}$ as a nonnegative quantity. With this in mind, let us rewrite the energy expression (7) in terms of the starred variables:

$E_{f}=\int_{0}^{t_{f}} \sum_{i=1}^{4}\left(I \dot{\Omega}_{i}^{*}(t) \Omega_{i}^{*}(t)+\left(d \Omega_{i}^{*}(t)+d_{v}\right) \Omega_{i}^{* 2}(t)\right) d t$

From (20) and (23) we write

$\bar{E}_{f} \doteq \int_{0}^{t_{f}} \sum_{i=1}^{4} I \dot{\Omega}_{i}^{*}(t) \Omega_{i}^{*}(t) d t=\frac{1}{2} I K^{-1}[1,1,1,1] \int_{0}^{t_{f}}\left[\begin{array}{l}\dot{U}_{b}^{*} \\ \dot{M}_{b}^{*}\end{array}\right] d t$

$=\frac{1}{2} I K^{-1}[1,1,1,1]\left[\begin{array}{c}U_{b}^{*}\left(t_{f}\right)-U_{b}^{*}(0) \\ M_{b}^{*}\left(t_{f}\right)-M_{b}^{*}(0)\end{array}\right]$
But observing (9) and (12) and recalling (17)-(18) and the fact that the initial and final linear and angular accelerations are all zeros we have (see (9) and (12)) $U_{b}^{*}(0)=U_{b}^{*}\left(t_{f}\right)=g$ and at the end points the torque vector satisfies $M_{b}^{*}(0)=M_{b}^{*}\left(t_{f}\right)=0$ and (23) becomes

$\bar{E}_{f}=0$

Hence, (22) reduces to

$$
\left.E_{f} \doteq \int_{0}^{t_{f}} \sum_{i=1}^{4}\left(d \Omega_{i}^{*}(t)+d_{v}\right) \Omega_{i}^{* 2}(t)\right) d t
$$

and the control objective is to minimize (26) subject to (21) and $\ddot{z}^{*}(t)+\mathrm{g}>0$, where $\Omega_{i}^{*}, i=1,2,3,4$ are functions of the flat output (as a result of equations (20), (12), and (9)). Hence, we have $\Omega_{i}^{*}=\Omega_{i}^{*}\left(a_{i}, b_{i}, c_{i}\right)$ and observing (26) we write $E_{f}=E_{f}\left(a_{i}, b_{i}, c_{i}\right)$. The bottom line is that the original optimal control problem has been reformulated to a simplified nonlinear dynamic programming problem. Methods of dynamic programming [13], [14] have found applications in numerous fields. The application of nonlinear dynamic programming to the optimal control problem in the considered quadrotor model is currently under study. However, for the sake of illustration we will demonstrate the approach for a simple case.

Note that once we have $\Omega_{i}^{*}(\mathrm{t}), i=1,2,3,4$ it is possible to determine by the second equation of (5) the terms $\tau_{i}^{*}(t)=k_{t} i_{i}^{*}(t)$, and then the input signals $v_{i}^{*}(t), i=1,2,3,4$ can be evaluated by the first equation.

\section{Example}

Although, as noted above, the optimal control problem has been simplified by the algorithm based on the flatness property, the nonlinearity and the complexity of the system still make it numerically challenging. We will present here an elementary example which demonstrates the approach of the considered problem. (A wide development of a suitable algorithm is now under further study). The relevant physical parameters of the quadrotor in the example are as follows. The quadrotor mass is $m=1[k g]$, the thrust and drag coefficients are $b=9.5 \times 10^{-3}\left[\mathrm{~N} /(\mathrm{rad} / \mathrm{sec})^{2}\right] \& d=7.5 \times 10^{-4}\left[\mathrm{Nm} /(\mathrm{rad} / \mathrm{sec})^{2}\right]$, and the gravity acceleration is $g=9.81\left[\mathrm{~m} / \mathrm{sec}^{2}\right]$.

Regarding the actuator parameters we assume a small DC motors and ignore the inductance $L$, the resistance is $R=0.2[\Omega]$ and $k_{t}=2.3 \times 10^{-2}[\mathrm{Nm} / \mathrm{A}]$ is the torque constant. The viscous damping coefficient is taken as $d_{v}=0.01[\mathrm{Nm} /(\mathrm{rad} / \mathrm{sec})]$, the electromotive constant in (5) is $k_{v}=100[(\mathrm{rad} / \mathrm{sec}) / v]$, and the rotor inertia (including the propeller) is $I=5 \times 10^{-5}\left[\mathrm{kgm}^{2}\right]$.

In the current simple example, we assume a vertical flight i.e. $x^{*}(t)=y^{*}(t)=0$, for all $0 \leq t \leq t_{f}$. This implies 
$\xi^{*}(t)=\left[\phi^{*}(\mathrm{t}), \theta^{*}(\mathrm{t}), \psi^{*}(t)\right]^{T}=0 \quad$ and $\quad M_{b}^{*}(t)=0$ for all $0 \leq t \leq t_{f}$. From (19) and the first equation in (20) one arrives at

$\left[\Omega_{1}^{* 2}, \Omega_{1}^{* 2}, \Omega_{1}^{* 2}, \Omega_{1}^{* 2}\right]^{T}=K^{-1}\left[\begin{array}{c}U_{b}^{*} \\ 0\end{array}\right]=\frac{1}{4 b}\left[U_{b}^{*}, U_{b}^{*}, U_{b}^{*}, U_{b}^{*}\right]^{T}$

and since we take $m=1,(26)$ becomes

$E_{f}=\int_{0}^{t_{f}} \sum_{i=1}^{4}\left(d \sqrt{U_{b}^{*}(t)} / 4 b+d_{v} / 4 \mathrm{~b}\right) U_{b}^{*}(t) d t$

and recalling the numerical data

$E_{f}=\int_{0}^{t_{f}} 4\left(1.79 U_{b}^{*}(t)^{3 / 2}+23.6 U_{b}^{*}(t)\right) d t$

From the flatness property and the fact that $x^{*}(t)=y^{*}(t)=0$, for all $0 \leq t \leq t_{f}$ we have

$U_{b}^{*}(t)=\ddot{\mathrm{Z}}^{*}(t)+g>0$

Recalling (21) and (22) let $z^{*}(t)=\sum_{i=3}^{n_{3}} c_{i} t^{i}$. The selected final time is $t_{f}=5[\mathrm{sec}]$ and the desired final position is

$\varphi_{1 f}{ }^{*}=[0,0,10]^{T}$. We consider the case $\operatorname{deg}\left\{z^{*}(t)\right\}=n_{z}=6$. Using (21) for $n_{z}=6$ the coefficients $c_{i}, i=3,4,5,6$ must be selected such that the polynomial $z^{*}(t)=\mathrm{c}_{3} t^{3}+\mathrm{c}_{4} t^{4}+\mathrm{c}_{5} t^{5}+\mathrm{c}_{6} t^{6}$ satisfies the 3 conditions

$z^{*}\left(t_{f}\right)=\mathrm{c}_{3} t_{f}^{3}+\mathrm{c}_{4} t_{f}^{4}+\mathrm{c}_{5} t_{f}^{5}+\mathrm{c}_{6} t_{f}{ }^{6}=10$

$\dot{z}^{*}\left(t_{f}\right)=3 \mathrm{c}_{3} t_{f}{ }^{2}+4 \mathrm{c}_{4} t_{f}^{3}+5 \mathrm{c}_{5} t_{f}^{4}+6 \mathrm{c}_{6} t_{f}^{5}=0$

$\ddot{z}^{*}\left(t_{f}\right)=6 \mathrm{c}_{3} t_{f}+12 \mathrm{c}_{4} t_{f}^{2}+20 \mathrm{c}_{5} t_{f}^{3}+30 \mathrm{c}_{6} t_{f}^{4}=0$

and $\ddot{z}^{*}(t)+\mathrm{g}>0, \forall t \in\left[0, t_{f}\right]$.

We shall compute a set of admissible constants (a set of constants $c_{i}, i=3,4,5,6$ that satisfy the listed conditions) that minimize an upper bound to $E_{f}$. From (29) $E_{f}=E_{f 1}+E_{f 2}$ with

$E_{f 1} \doteq \int_{0}^{t_{f}} 7.16\left(U_{b}^{*}(t)\right)^{3 / 2} d t, E_{f 2} \doteq \int_{0}^{t_{f}} 94.4 U_{b}^{*}(t) d t$

Now, since (see (14)-(15)) $\dot{z}^{*}(0)=\dot{z}^{*}\left(t_{f}\right)=0$ we have using (30)

$$
\begin{aligned}
E_{f 2} & \doteq 94.4 \int_{0}^{t_{f}} U_{b}{ }^{*}(t) d t \\
& =94.4 \int_{0}^{t_{f}}\left(\ddot{z}^{*}(t)+g\right) d t=94.4 g t_{f}
\end{aligned}
$$

Next, we consider the term $E_{f 1}$ in (32). For $\ddot{z}^{*}(t)+\mathrm{g}>0$ we have using Schwartz's inequality

$$
\begin{aligned}
E_{f 1} & \doteq 7.16 \int_{0}^{t_{f}} U_{b}^{*}(t)^{3 / 2} d t=7.16 \int_{0}^{t_{f}} U_{b}^{*}(t) U_{b}^{*}(t)^{1 / 2} d t \\
& \leq 7.16\left[\int_{0}^{t_{f}}\left(\ddot{z}^{*}(t)+\mathrm{g}\right)^{2} d t\right]^{1 / 2}\left[\int_{0}^{t_{f}}\left(\ddot{z}^{*}(t)+\mathrm{g}\right) d t\right]^{1 / 2}
\end{aligned}
$$

Recalling (34) and using $\left(\ddot{z}^{*}+\mathrm{g}\right)^{2}=\ddot{z}^{* 2}+2 \ddot{z}^{*} \mathrm{~g}+\mathrm{g}^{2}$ we get

$$
E_{f 1} \leq 7.16 \sqrt{g t_{f}}\left[\int_{0}^{t_{f}} \ddot{z}^{*}(t)^{2} d t+g^{2} t_{f}\right]^{1 / 2}
$$

Hence, our current objective is to compute an admissible set of coefficients $c_{i}, i=3,4,5,6$ that minimizes the right-hand side of (35). Let

$$
f\left(t_{f}, c\right) \doteq \int_{0}^{t_{f}} \ddot{z}^{*}(t)^{2} d t
$$

where $c=\left[c_{3}, c_{4}, c_{5}, c_{6}\right]^{T}$ is the coefficients vector. We have $f\left(t_{f}, 0\right)=0$ and $f\left(t_{f}, c\right)>0, \forall c \neq 0, t_{f}>0$, and $f\left(\mathrm{t}_{f}, c\right)=\mathrm{c}^{T} Q\left(t_{f}\right) c / 2$ where $Q\left(t_{f}\right)=Q^{T}\left(t_{f}\right) \in R^{4 \times 4}$ is a positive definite matrix. Hence, the problem of minimizing $f\left(t_{f}, c\right)$ subject to the constraint (31) might be obtained by using tools from the theory of the quadratic constrained minimization problems [15].

To this end we can rewrite (31) as $H\left(t_{f}\right) c=a$ where

$$
H\left(t_{f}\right)=\left[\begin{array}{cccc}
t_{f}^{3} & t_{f}{ }^{4} & t_{f}{ }^{5} & t_{f}{ }^{6} \\
3 t_{f}{ }^{2} & 4 t_{f}{ }^{3} & 5 t_{f}{ }^{4} & 6 t_{f}{ }^{5} \\
6 t_{f} & 12 t_{f}{ }^{2} & 20 t_{f}{ }^{3} & 30 t_{f}{ }^{4}
\end{array}\right], a=\left[\begin{array}{c}
10 \\
0 \\
0
\end{array}\right]
$$

It is easy to check that the matrix $H\left(t_{f}\right), t_{f}>0$ is full row rank. Hence, the minimum of $f\left(\mathrm{t}_{f}, c\right)$, subject to $H c=a$ is unique and can be determined by

$$
\left[\begin{array}{cc}
Q\left(t_{f}\right) & H\left(t_{f}\right)^{T} \\
H\left(t_{f}\right) & 0
\end{array}\right]\left[\begin{array}{l}
c \\
\lambda
\end{array}\right]=\left[\begin{array}{l}
0 \\
a
\end{array}\right]
$$

where $\lambda \in R^{3}$ is the Lagrange multipliers vector. Since the $Q$ is positive definite and $H$ is of full row rank, the matrix in (38) is invertible. For $t_{f}=5$ we obtain the following numerical results. The coefficients vector $c$ is $c=\left[c_{3}, c_{4}, c_{5}, c_{6}\right]^{T}=\left[0.79736,-0.23842,1.8884 \times 10^{-2}, 2.1099 \times 10^{-5}\right]^{T}$ 
and functions $z(t)$ (see Fig. 3) and $\ddot{z}(t)$ are given by

$$
\begin{aligned}
z^{*}(t) & =0.79736 t^{3}-0.23842 t^{4} \\
& +1.8884 \times 10^{-2} t^{5}+2.1099 \times 10^{-5} t^{6} \\
\ddot{z}^{*}(t) & =4.7842 t-2.861 t^{2}+0.37768 t^{3} \\
& +6.3297 \times 10^{-4} t^{4}, \quad t \in[0,5]
\end{aligned}
$$

Further, $f(5, c)=\mathrm{c}^{T} Q(5) c / 2=13.719$ and hence, (35) and (36) yield $\quad E_{f 1} \leq 7.16 \sqrt{5 g}\left[13.719+5 g^{2}\right]^{1 / 2}=1115.6$ and from (32) $E_{f 2}=94.4 g 5=4630.3$ and finally

$$
\begin{aligned}
E_{f}=E_{f 1} & +E_{f 2} \leq 1115.6+4630.3=5745.7[\mathrm{~J} .] . \text { Using (30) } \\
U_{b}^{*}(t)= & 4.7842 t-2.861 t^{2}+0.37768 t^{3} \\
& +6.3297 \times 10^{-4} t^{4}+g>0, t \in[0,5]
\end{aligned}
$$

(see Fig. 4) and from (27) and (19) the motor speed is $\Omega_{i}^{*}(t)=\left[U^{*}(t) /(4 \mathrm{~b})\right]^{1 / 2}$

$$
\begin{aligned}
& =\left[503.94 t-301.36 t^{2}+39.783 t^{3}\right. \\
& \left.+6.6673 \times 10^{-2} t^{4}+1033.3\right]^{1 / 2}, t \in[0,5]
\end{aligned}
$$

From (5) $\quad k_{t} i(t)=\tau(t)=I \dot{\Omega}_{i}(t)+d \Omega_{i}^{2}(t)+d_{v} \Omega_{i}(t)$, and using the numerical data we have $i(t)=2.174 \times 10^{-3} \dot{\Omega}_{i}(t)+3.043 \times 10^{-2} \Omega_{i}^{2}(t)+0.435 \Omega_{i}(t)$ and from the first equation of (5) we arrive at $v_{i}(t)=R i(t)+\Omega_{i}(t) / k_{v}=0.2 i(t)+0.01 \Omega_{i}(t)$.

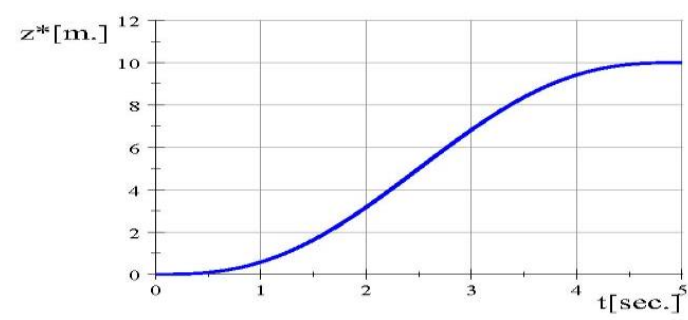

Figure 3. Time history plot of the vertical position $z^{*}(t)$.

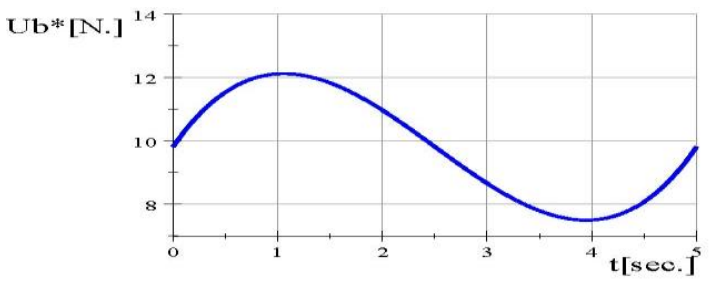

Figure 4. Time history plot of the thrust force $U_{b}{ }^{*}(t)$.

\section{Conclusion}

Based on the concept of flatness in nonlinear systems theory a minimum energy consumption control algorithm is applied to motion control of quadrotor-type helicopters. Energy saving will extend the flight time of the aircraft and hence, will allow for more flexible and effective supervision and inspection of power utility assets. Further research is under way to expand the dynamic programming algorithm to solve the optimal control problem for the complete nonlinear model of the system under consideration in case of model uncertainties. The mathematical procedure in this article is based on the use of polynomial functions but to reduce oscillations between data points, a more suitable set of linear independent functions should be considered.

\section{References}

[1] A. Hand, "Drones speed solar and wind farm inspection" https://www.automationworld.com/article/technologies/mai ntenance-reliability/drones-speed-solar-and-wind-farminspection, March 7, 2018.

[2] C. Eschmann, C.-M. Kuo, C.-H. Kuo, C. Boller, Highresolution multisensor infrastructure inspection with unmanned aircraft systems", International Archives of the Photogrammetry, Remote Sensing and Spatial Information Sciences, Vol. XL-1/W2, UAV-g, Rostock, Germany, September 4-6, 2013.

[3] S. Montambault, J. Beaudry, K. Toussaint and N. Pouliot, "On the application of VTOL UAVs to the inspection of power utility assets Hydro-Québec", 1st International Conference on Applied Robotics for the Power Industry Delta Centre-Ville, October 5-7, 2010.

[4] K. Fehrenbacher, "How drones are lowering the cost of clean energy", https://www.greenbiz.com/article/howdrones-are-lowering-cost-clean-energy, March 15, 2018.

[5] J. Pyper, "Why Drones Are 'Game-changing' for renewable energy"

https://www.greentechmedia.com/articles/read/why-dronesare-game-changing-for-renewable-energy\#gs.bWRGJig, July 15, 2016.

[6] A. C. Satici, H. Poonawala1, and M. W. Spong, "Robust Optimal Control of Quadrotor UAVs", IEEE Access, Vol. 1, pp. 79-83, 2013.

[7] F. Morbidi, R. Cano, and D. Lara, "Minimum-energy path generation for a quadrotor UAV", IEEE Int. Conf. on Robotics \& Automation, Stocholm, Sweden, May 2016.

[8] F. Yacef, N. Rizoug, O. Bouhali, and M. Hamerlain, "Optimization of Energy Consumption for Quadrotor UAV", Proc. Of the Int. Micro Air Vehicle Conf. and Flight Competition, pp. 215-222, Sept. 18-21, Toulouse 2017.

[9] A, Ailon and S. Arogeti, "Closed-form nonlinear tracking controllers for quadrotors with model and input generator uncertainties," Automatica, Vol. 54, pp. 317-324, 2015.

[10] M. Fliess, J. L. Levine, P. Martin, \& P. Rouchon, "Flatness and defect of non-linear systems: introductory theory and examples. Int. J. Control, Vol. 61, pp. 13271361, 1995.

[11] M. Turpin, N. Michael, \& V. Kumar, "Trajectory design and control for aggressive formation flight with quadrotors", Autonom. Robots, Vol. 33, pp. 143-156, 2012.

[12] K. Eliker, G. Zhang, S. Grouni, \& W. Zhang, “An optimization problem for quadrcopter reference flight trajectory generation", Hindawi, J. of Advance Transportation, Vol 2018, ID 6574183, pp. 1-15, 2012.

[13] S. Danø, Nonlinear and Dynamic Programming, Springer Verlag, Wien, 1975.

[14] R. D. Robinett, III, D. G. Wilson, G. R. Eisler, J. E. Hurtado, Applied Dynamic Programming for Optimization of Dynamical Systems, Siam Series: Advances in Design and Control, 2005.

[15] J. Gallier, Geometric Methods and Applications, Second Edition, Springer, New York, 2011. 